\title{
Intermédialités
}

Histoire et théorie des arts, des lettres et des techniques

Intermediality

History and Theory of the Arts, Literature and Technologies

\section{Un théâtre d'acteurs vidéographiques : Les aveugles de Denis Marleau}

\section{Hélène Jacques}

Numéro 6, automne 2005

Remédier

Remediation

URI : https://id.erudit.org/iderudit/1005507ar

DOI : https://doi.org/10.7202/1005507ar

Aller au sommaire du numéro

Éditeur(s)

Centre de recherche sur l'intermédialité

ISSN

1705-8546 (imprimé)

1920-3136 (numérique)

Découvrir la revue

Citer cet article

Jacques, H. (2005). Un théâtre d'acteurs vidéographiques : Les aveugles de Denis Marleau. Intermédialités / Intermediality, (6), 79-94.

https://doi.org/10.7202/1005507ar
Résumé de l'article

Denis Marleau conçoit, dans sa mise en scène de la pièce Les aveugles, de Maeterlinck, un dispositif scénique évoquant le procédé de la fantasmagorie, dans lequel l'acteur est remplacé par des masques et des projections vidéographiques. Ce texte aborde l'intégration des autres arts sur les scènes de théâtre, de même qu'il traite des conséquences des échanges interartistiques sur la définition de l'oeuvre théâtrale à sa réception. Le processus de création des Aveugles et les techniques du langage vidéo empruntées par le metteur en scène sont étudiés en fonction de l'approche " ethnographique " d'Antoine Hennion qui, pour analyser l'objet musical, observe les moyens techniques et humains mis à contribution dans l'élaboration de l'oeuvre.
Ce document est protégé par la loi sur le droit d'auteur. L'utilisation des services d'Érudit (y compris la reproduction) est assujettie à sa politique d'utilisation que vous pouvez consulter en ligne.

https://apropos.erudit.org/fr/usagers/politique-dutilisation/ 


\title{
Un théâtre d'acteurs vidéographiques : Les aveugles de Denis Marleau'
}

\author{
Hélène Jacques
}

«[L]e théâtre que je fais, [affirme Denis Marleau, en 1992], cherche aussi le moyen d'accéder à une plus grande musicalité2. » Durant les années 1980, le travail du directeur d'UBU s'apparente en effet à celui d'un chef d'orchestre, en ce sens qu'il fait entendre les textes aux allures de partition musicale des Schwitters, Queneau, Maïakowski et autres auteurs du répertoire des avant-gardes littéraires dans lequel il puise pour élaborer ses spectacles-collages. Dans les premières créations où dominent ludisme et dérision, où est offerte "une interrogation sur la langue, sa logique et ses surprises ${ }^{3}$ ", les acteurs accordent une attention particulière aux rythmes, tonalités et intensités des textes qu'ils prononcent souvent en chœur, leur conférant une sonorité précise. Cette prééminence accordée à la référence musicale et au travail vocal chez Marleau - qui demeure jusque dans ses créations récentes ${ }^{4}-$, met en lumière ce qui définit, en partie, l'acte

1. Ce texte a d'abord été écrit dans le cadre du séminaire Littérature et médias, dirigé par Philippe Despoix au Département de littérature comparée de l'Université de Montréal.

2. Josette Féral, «Je sentais de plus en plus que je ne deviendrais pas un acteur. Entretien avec Denis Marleau », Jeu, nº 62, 1992, p. 112.

3. Yannic Mancel, «D’UBU à Woyzeck: pour une relecture des avant-gardes », JeanFrançois Chassay (dir.), L'album du théâtre UBU, Montréal, Carnières, Cahiers de théâtre Jeu, Éditions Lansman, 1994, p. 14.

4. Denis Marleau écrivait récemment que «ce qui [lui] importe au théâtre, c'est d'arriver à mettre en espace les sonorités du texte comme une matière concrète à travers la profération de l'acteur. » (Denis Marleau et Stéphanie Jasmin, «La non-action comme terrain fertile pour un travail d'acteur. Témoignage », L'Annuaire théâtral, $\mathrm{n}^{\circ} 36$, automne 2004, p. 101) 
théâtral, à l'instar de l'objet musical: arts du temps, tous deux sont également éphémères.

Antoine Hennion s'interroge sur la difficulté d'analyser la musique et souligne, dans La passion musicale. Une sociologie de la médiation, que l'objet musical ne possède pas, contrairement à l'œuvre d'art plastique, de matière propre, «n'est pas une chose posée devant soi »; « l'auditeur n’a pas l'œuvre en face de lui, saisissable d'un coup, mais une durée, qu'il ne peut comprendre que s'il s'y abandonne - adieu la distance critique - , et commenter que s'il en est sorti, à travers la reconstruction de sa mémoire5 ». Face à une œuvre théâtrale, si le spectateur a bel et bien devant lui un espace rempli d'objets et d'acteurs, s'il peut se procurer le texte publié, lors de la représentation, néanmoins, il écoute et regarde le spectacle «vivant» sans pouvoir en interrompre le cours ni revenir en arrière: il est confronté, comme l'auditeur de musique, à une durée - et plus précisément à un mouvement - qu'il ne peut manipuler. D'autre part, dans de nombreuses représentations théâtrales, exception faite de celles qui jouent explicitement avec les codes pour briser l'illusion, le dispositif technique sur lequel repose le spectacle est minutieusement dissimulé pour se faire oublier et ce, afin que le spectateur «s'abandonne» à la proposition scénique. Étudier un spectacle théâtral consiste à se remémorer ce qui en principe était caché, ce qui devait être invisible pour que prenne forme, émerge l'univers fictionnel. Il s'avère par conséquent quasi impossible d'analyser la représentation théâtrale autrement qu'à partir du point de vue, circonstanciel, du spectateur d'un soir devant se souvenir de l'expérience vécue. Bien entendu, la représentation peut être filmée, mais la copie vidéo ne constitue qu'un piètre duplicata, en ce sens que l'environnement sonore, souvent englobant, de même que la subtilité des éclairages se perdent presque complètement dans la reproduction électronique.

Si le spectateur ou l'auditeur doit faire son deuil de pouvoir rendre compte rigoureusement de son expérience, il peut cependant s'appuyer sur les «traces » de l'œuvre, car selon Antoine Hennion, derrière chaque œuvre musicale - et dans le cas qui nous intéresse, on le suppose, derrière chaque œuvre théâtrale «se profile toujours l'appui privilégié sur une technique particulière de fixation, de mise en objet» : il est par conséquent concevable que l'étude des moyens de production d'une œuvre donne l'occasion de la comprendre «à travers ses dispo sitifs intermédiaires». (PM, p. 298) Hennion propose d'analyser «la construction

5. Antoine Hennion, La passion musicale. Une sociologie de la médiation, Paris, Métailié, 1993, p. 286. Désormais, les références à cet ouvrage seront indiquées par le sigle « PM » suivi de la page et placées entre parenthèses dans le corps du texte. 
même de l'objet musical » (PM, p. 289), plutôt que de choisir entre une approche réduisant la musique à la partition ou celle la considérant comme essence pure, immatérielle. Il s'intéresse aux «objets techniques, [aux] supports matériels, [aux] instruments sur lesquels s'appuie l'art pour durer» (PM, p. 297) afin d'analyser les genres musicaux, puisque ces supports «n’obéissent pas aux différentes genres, ils les produisent» (PM, p. 3O2). Grâce à une approche empruntant à la sociologie et à l'anthropologie, il analyse la musique comme une «mobilisation active d'une foule de participants, hommes et choses, instruments et écrits, lieux et dispositifs. » (PM, p. 290) Dans un ouvrage qu'il publie en 1981, Les professionnels du disque. Une sociologie des variétés, Hennion explore déjà cette avenue en analysant la production discographique à partir «du contenu même du travail des producteurs de variétés ${ }^{\star} »$. Suite aux observations faites sur le terrain dans de nombreuses firmes de production de disques, Hennion décrit le travail de tous les collaborateurs (du directeur à l'ingénieur du son en studio, en passant par l'interprète et le parolier) et définit chacune des étapes de production d'une chanson. Cette «ethnographie» du travail de production lui permet de faire la genèse de la chanson sous l'angle de sa fabrication et de saisir comment fonctionne et se construit le succès, enjeu primordial de l'industrie des variétés.

L'étude proposée ici est bien sûr plus modeste que celle d'Hennion et portera sur un spectacle de la compagnie de création UBU : Les aveugles, fantasmagorie technologique, pièce que Denis Marleau a mise en scène au Musée d'art contemporain de Montréal en 2002. Cette création, multidisciplinaire, a la particularité de déplacer le concept de théâtralité, dans la mesure où l'acteur, absent de la scène, est remplacé par des projections vidéographiques. En m’interrogeant, à l'instar d'Antoine Hennion, sur les conditions d'émergence de l'œuvre, en identifiant les principes de construction propres aux différentes disciplines qui concourent à sa production, je suggère qu'il sera possible d'observer la manière dont la vidéo participe à la conception d'une forme théâtrale nouvelle. Cette forme, du reste, s'inscrit dans une recherche amorcée en 1997, alors que Marleau, dans l'adaptation d'un récit d'Antonio Tabucchi, Les trois derniers jours de Fernando Pessoa, a réalisé un dispositif intégrant des projections vidéographiques pour représenter les hétéronymes du poète portugais. Le visage filmé de l'acteur interprétant Pessoa, Paul Savoie, était projeté sur différents supports, dont un masque

6. Antoine Hennion, Les professionnels du disque. Une sociologie des variétés, Paris, Métailié, 1981, p. 12. 
porté par un acteur muet?. La réflexion sur les questions du double, de l'identité, de la représentation de l'invisible, se poursuit dans Les aveugles, où le même procédé, perfectionné, est utilisé. La forme conçue, en outre, répond en écho, un siècle plus tard, aux idées de Maurice Maeterlinck, qui envisage la scène comme un espace de suggestion et propose l'emploi d'objets symboliques (la marionnette, le masque, la figure de cire) pour représenter l'homme et la mort. La poétique du fantôme de Maeterlinck se voit réactualisée par des moyens scéniques auxquels le dramaturge belge ne pouvait, bien entendu, référer. Le metteur en scène évoque également, dans le sous-titre, la fantasmagorie, spectacle très en vogue au XIX ${ }^{\mathrm{e}}$ siècle, au cours duquel un illusionniste faisait apparaître, grâce à un système éclairant un objet de verre, des spectres et des figures. Lappareillage, chez Marleau, dans une opération s'apparentant à une remédiation, devient technologique.

\section{THÉÂTRE MULTIDISCIPLINAIRE}

La convocation des autres arts sur les scènes de théâtre, tant au Québec qu'à l'étranger, acquiert une importance accrue depuis une trentaine d'années ${ }^{8}$. Résultant d'une opération de métissage des disciplines et des médias, de nombreuses œuvres - dont Les aveugles, théâtre présenté au musée, «sculpture vidéographi$q^{q u} e^{9} »-$, font éclater les frontières disciplinaires. Selon Marie-Christine Lesage, le théâtre multidisciplinaire «met en place une structure communicationnelle dynamique au sein de laquelle les arts convoqués gardent leurs propriétés, tout en étant soumis à un système de représentation différent de celui qui régit les composantes de ces arts de façon indépendante ${ }^{1 \circ}$. "Ce théâtre, poursuit-elle, modifie les conditions de perception de l'œuvre et «met de l'avant une complexité nouvelle ${ }^{11}$ ». Mais dans la mesure où le théâtre, au fondement, émerge de l'assemblage de multiples médias et que le rapport entre les différentes disciplines

7. Dans Les trois derniers jours de Fernando Pessoa (1997), puis Urfaust (1999), Les aveugles (2002), et plus récemment dans Dors mon petit enfant et Comédie (2004), des visages filmés sont projetés sur des masques selon le même procédé.

8. Je renvoie, pour un panorama de ce phénomène au Québec, à l'article de MarieChristine Lesage, qui analyse plusieurs pratiques comme autant de cas de figure: «Le théâtre et les autres arts. Matériaux composites », dans Dominique Lafon (dir.), Le théâtre québécois. 1975-1995, Montréal, Fides, coll. «Archives des lettres canadiennes», 2001, Tome X, p. 335-360.

9. L'expression est de Gilles Daigneault, «À propos de quelques jumelages heureux", Espace 60, été 2002, p. 27.

10. Marie-Christine Lesage, «Le théâtre et les autres arts », p. 337.

11. Marie-Christine Lesage, "Le théâtre et les autres arts», p. 360. 
(et le texte) n'a eu de cesse de changer au cours de l'histoire ${ }^{12}$, il importe de s'interroger sur la spécificité même du phénomène contemporain d'hybridation des formes théâtrales, et sur ses conséquences quant aux esthétiques développées et à la réception des œuvres. Qu'est-ce qui différencie, en d'autres termes, l'œuvre multidisciplinaire de la représentation théâtrale à laquelle on n'attribue pas cette dénomination?

Denis Marleau, pour sa part, revendique le caractère multidisciplinaire de sa démarche, à tout le moins si l'on se fie aux propos qu'il a tenus en 1998: dans la mesure où les avant-gardes historiques ont intéressé le metteur en scène pendant les années 1980, et que certains de leurs procédés de création ont constitué une source d'inspiration, il a été «entraîné, en somme, à "désétanchéifier" [sa] pratique du théâtre, en nouant des collaborations artistiques avec des musiciens, des danseurs, des peintres et des sculpteurs ${ }^{13}$ ». Les frontières de l'acte théâtral éclatent puisque sur la scène d'UBU, «le théâtre et la peinture se rencontrent, et au moins par deux fois ", affirme Olivier Asselin. D’une part, "parce que le Théâtre UBU s'interroge surtout sur l'avant-garde [, alors que] de nombreux artistes - peintres et écrivains en particulier — vont [...] monter sur scène » et que, plusieurs créations ayant été présentées dans des musées, «le Théâtre UBU a régulièrement été associé à l'institution muséale ${ }^{14}$ ». À l'instar des artistes multidisciplinaires des avant-gardes, Marleau «désétanchéifie» sa pratique, en convoquant sur scène arts visuels, musique, danse et, plus récemment, art vidéo. Marie-Christine Lesage s'est aussi intéressée au métissage des arts chez UBU, et plus particulièrement à la collaboration de Marleau avec des sculpteurs et des plasticiens (Pierre Granche, Michel Goulet, Zaven Paré), laquelle a donné lieu à la conception d'espaces de jeu complexes et hybrides, des «installation[s] scénique [s], à mi-chemin entre l'installation en art visuel et la scénographie ${ }^{15}$ ». Ainsi, l'emprunt à l'installation permet d'élaborer des scénographies inédites et

12. Pensons aux visions esthétiques du siècle dernier, du «Gesamtkunstwerk» de Wagner à la «collaboration à distance» de Brecht. Voir Béatrice Picon-Vallin, «La mise en scène et le texte », Christian Doumet et al. (dir.), L'art et l'hybride, Paris, Presses universitaires de Vincennes, 2001, p. 103-116.

13. Josette Féral, «Denis Marleau: une approche ludique et poétique», dans Mise en scène et jeu de l'acteur, Montréal, Cahiers de théâtre Jeu, Carnières, Lansman Éditeur, 1998 , tome II, p. 185 .

14. Olivier Asselin, «Le Théâtre UBU. L'avant-garde entre la scène et le musée», Parachute, $n^{\circ} 66$, avril-mai-juin 1992, p. 20 et 22.

15. Marie-Christine Lesage, «Installations scéniques. Le cas du Théâtre UBU et du collectif Recto-Verso », L’Annuaire théâtral, n 26, automne 1999, p. 32. 
de réfléchir à de nouvelles façons de présenter l'espace en «travaill[a]nt le champ

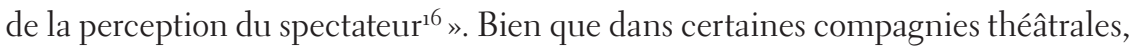
lorsque de telles collaborations ont cours, le rôle du metteur en scène se trouve bouleversé ${ }^{17}$, quoique l'on reconnaisse dans les installations scéniques chez UBU des éléments du langage visuel des artistes (par exemple, la pyramide tronquée de Pierre Granche dans Luna Park ${ }^{18}$ ), Denis Marleau se présente généralement en tant qu'auteur unique du spectacle et est présenté comme tel par la critique. Si les différents langages artistiques intégrés sur la scène d'UBU complexifient la représentation, font éclater la notion de scénographie en tant qu'illustration de l'espace dramatique, déplacent la notion de théâtralité comme dans Les aveugles, ils trouvent néanmoins leur cohérence dans la vision unique du metteur en scène, dont la source est habituellement le texte dramatique.

\section{AUTOUR DES AVEUGLES}

\section{La fantasmagorie}

Les douze aveugles de Maurice Maeterlinck, perdus dans une forêt, attendent le retour du guide devant les ramener à la pension où ils habitent. Effrayés par l'environnement hostile qui les entoure, ils restent immobiles et s'échangent quelques paroles inquiètes avant de réaliser que le guide est là, parmi eux, mort depuis plusieurs heures. Se sachant condamnés, ils attendent désormais la mort qu'ils écoutent avec frayeur se rapprocher: au cœur du drame se trouve ainsi la question de l'angoisse métaphysique de l'homme confronté à l'idée de sa disparition, et le problème de la représentation de l'invisible. Tant dans ses œuvres dramatiques que dans ses essais, Maeterlinck développe une poétique du fantôme, où la suggestion de l'invisible et de la mort devient un enjeu fondamental.

16. Marie-Christine Lesage, «Installations scéniques», p. 43.

17. C'est le cas chez Recto-Verso, où «l'installateur scénique contamine le théâtre par sa propre pratique artistique, il fait de l'espace scénique une matière autonome qui peut se lire comme une œuvre visuelle relativement indépendante de la mise en scène. » (Marie-Christine Lesage, «Installations scéniques», p. 32) D’autre part, le metteur en scène peut aussi collaborer étroitement avec des concepteurs qui deviennent du coup cocréateurs. Aux Deux Mondes, par exemple, le metteur en scène Daniel Meilleur affirme travailler, pour certains projets «simultanément avec un compositeur de musique, un vidéaste, un auteur [...]. Tous ces créateurs “écrivent” le spectacle.» (Daniel Meilleur, «Le risque de la beauté et de la création », Jeu, n ${ }^{\circ} 116$, mars 2005, p. 101)

18. Lola Noël et Louise Vigeant, «Encadrements et pyramides. Pierre Granche chez UBU », Jeu, no 63, février 1992, p. 31-39. 
Proposant la disparition de l'acteur des scènes de théâtre, le poète belge affirmait en 1890 , dans une formule souvent reprise, qu'

il faudrait peut-être écarter entièrement l'être vivant de la scène. Il n'est pas dit qu'on ne retournerait pas ainsi vers un art des siècles très anciens, dont les masques des tragiques grecs portent peut-être les dernières traces. [...] L'être humain sera-t-il remplacé par une ombre, une projection de formes symboliques ou un être qui aurait les allures de la vie sans avoir la vie ${ }^{19}$ ?

L'auteur de drames pour marionnettes attribue au masque et au pantin, instruments appartenant au théâtre de foire à la fin du XIX ${ }^{\mathrm{e}}$ siècle, un grand potentiel d'évocation. Il est probable, de plus, que la «projection de formes » à laquelle il renvoie soit inspirée des nombreuses inventions qui précèdent celle du cinématographe: différentes machineries, tels le «spectre de Robin» ou le «Pepper's Ghost», sont en effet utilisées sur les scènes et simulent la présence de spectres. Le poète symboliste, reconduisant certaines idées répandues au tournant du siècle (celles d'Edward Craig, entre autres exemples), désire dépasser la simple imitation de la nature à laquelle se limite la scène réaliste et représenter, par les moyens scéniques dont il constate l'émergence, l’invisible.

Le dispositif scénique conçu par Marleau, qui s'inscrit dans sa «recherche sur le double et le fantôme au théâtre ${ }^{20}$ ", semble alors constituer une forme de réponse aux désirs de Maeterlinck: l'homme en chair et en os est absent de sa mise en scène des Aveugles; il est représenté par « une projection de formes symboliques », soit des masques, animés par la vidéo, qui ont «les allures de la vie sans avoir la vie». Denis Marleau effectue aussi un rapprochement - sous forme d'allusion poétique - entre le dispositif scénique et la fantasmagorie, introduite en France en 1798 par Robertson, dont la visée est de créer l'illusion d'une présence. "Sorte de lanterne magique», le fantascope «se compose d'un système éclairant, d'un objet peint sur verre avec des couleurs transparentes et d'un objectif qui se déplace par rapport au verre peint. Tout l'appareil est monté sur un chariot que l'on peut rapprocher plus ou moins d'un écran translucide ${ }^{21}$.» Les spectateurs, plongés dans l'obscurité, «n'aperçoivent de l'écran que la partie qui reçoit l'image et, comme ils n'ont aucun point de comparaison, ils ne peuvent juger de la distance de cette image lumineuse; aussi, lorsque l'image grandira, ils

19. Maurice Maeterlinck, Euvres 1, Bruxelles, Éditions Complexes, 1999, p. 462.

20. Denis Marleau, «Les aveugles et l'utopie. À partir du projet d'une installationthéâtre ", Puck, n ${ }^{\circ} 13$, octobre 2000, p. 83.

21. Alexandre Joannis, «Fantascope », La grande encyclopédie. Inventaire raisonné des sciences, des lettres et des arts, Tome seizième, Paris, H. Lamirault, 1885-1902, p. 1197. 
croiront voir l'objet s'avancer vers $\mathrm{eux}^{22}$. » Lors de la représentation des Aveugles, le spectateur entre lui aussi dans une salle obscure et découvre, dispersés, douze masques lumineux qui semblent en suspension dans l'espace sombre. Les visages des deux acteurs, des projections vidéographiques sur douze masques-écrans, se réveillent alors et paraissent étrangement vivants: les lèvres remuent, les paupières battent, et les voix s'élèvent pour réciter les répliques laconiques de Maeterlinck. Le spectateur vit en somme une expérience semblable à celle du public des fantasmagories, le procédé conçu par Marleau rappelant les apparitions lumineuses et mouvantes que suscite le fantascope.

Dans la fantasmagorie, lorsque apparaissent des fantômes, «l'illusion produite est complète, et l'on comprend sans peine que dans des temps d'ignorance une pareille puissance d'évocation devait passer pour le résultat de quelque pacte infernal ${ }^{23}$.» Puisque l'écran est transparent et que le spectateur est dans l'obscurité, le dispositif reste dissimulé, ce qui concourt à l'illusion. Face aux masques animés des Aveugles, le spectateur, qui ne voit aussi que les supports illuminés, se pose de nombreuses questions quant au fonctionnement de la représentation: comme la fantasmagorie, en somme, le dispositif de Marleau provoque un effet de surprise, la vision offerte, celle de douze têtes apparemment animées et suspendues, étant très insolite. Enfin, la mise en scène d'UBU rappelle le caractère mystérieux de la fantasmagorie, que l'on accentuait autrefois «par des accessoires et la mise en scène: bruits de cloche, de chaînes, de tonnerre, émanations de fumées, etc. ${ }^{24}$ ». Chez UBU, des bruits de la forêt, composés de sons abstraits diffusés par des haut-parleurs, envahissent la salle et la scène, ce qui amplifie la sensation créée par la noirceur: masques et public baignent dans un univers commun, un espace inquiétant.

\section{Un aperçu du processus de création}

Un long processus de création, effectué dans le cadre d’un programme de résidence d'artiste organisé par le Musée d'art contemporain de Montréal, précède le spectacle. La description qui suit donne un aperçu très partiel de ce processus, dans la mesure où je n'y ai pas assisté : j’ai plutôt interrogé a posteriori la collaboratrice artistique du metteur en scène dans le projet, Stéphanie Jasmin, qui m’a révélé quelques grandes lignes du travail réalisé, dont le portrait reste cependant

22. Alexandre Joannis, «Fantascope», p. 1198.

23. Arthur Pougin, Dictionnaire historique et pittoresque du théatre et des arts qui s'y rattachent, Paris, Librairie de Firmin-Didot et $C^{\text {ie }}, 1885$, p. 356.

24. «Fantasmagorie », Grand Larousse universel, Paris, Larousse, 1989, tome 6, p. 4147. 
ici incomplet ${ }^{25}$. Au cours des étapes de la captation et du montage vidéo, Denis Marleau a travaillé avec deux acteurs, Paul Savoie incarnant les six rôles masculins de la pièce et Céline Bonnier, les six féminins. Les douze personnages ont été filmés un à un, en gros plan, l'acteur ayant la tête enserrée pour éviter les risques de mouvement et écoutant une répétitrice lui donner les répliques de tous les autres rôles. Le visage et la voix des acteurs ont donc été enregistrés en temps réel, et si Marleau n’a pas réalisé de montage majeur, il a cependant opéré des manipulations touchant la durée des répliques, les allongeant parfois ou les distillant, et a effectué quelques étirements de silences et contractions de temps morts, et ce, en respectant le texte et les indications de Maeterlinck. Ainsi, il a orchestré le rythme général, au fil de plusieurs écoutes, par des modifications subtiles qui ont aussi dû être rendues possibles à l'image tout en évitant le jump cut. Lors de la représentation, les douze captations vidéo sont projetées simultanément à partir de lecteurs DVD - dissimulés en régie - , sur autant de masques-écrans. Ces derniers ont été fabriqués par Claude Rodrigue, artiste sculpteur, à partir de masques de plâtre moulés sur les visages des deux acteurs. Les masques en matière plastique utilisés pour la représentation, légèrement plus grands que nature, constituent les supports, savamment disposés dans l'espace scénique par Denis Marleau selon les didascalies de Maeterlinck, sur lesquels sont projetées les images. L'ensemble de l'appareillage technique et les trépieds qui soutiennent les masques demeurent totalement invisibles dans la pénombre, de même que les faisceaux lumineux des projections, dont l'intensité est minutieusement contrôlée : les visages paraissent flotter dans l'espace.

La réalisation des Aveugles fait intervenir, sous la supervision du metteur en scène, créateur et concepteur du projet, le travail d'un sculpteur, celui de techniciens vidéastes et d'une conceptrice d'effets sonores. Diverses techniques et technologies sont utilisées : caméra, appareils de prise de son, ordinateurs, projecteurs DVD, etc., et l'une des particularités de ce spectacle réside dans le fait que les images vidéographiques remplacent non seulement les acteurs, mais aussi toutes les sources d'éclairage traditionnel. En effet, la lumière des projecteurs qui donne à voir, révèle sur la scène les acteurs, et le décor devient ici perceptible en soi et pour soi; plutôt que de soutenir la mise en scène au centre de laquelle se trouve l'acteur, les projections représentent l'essentiel du dispositif scénique.

25. Je remercie vivement Stéphanie Jasmin de m’avoir aidée dans la rédaction de cet article. Je renvoie en outre à un article de cette dernière, qui a témoigné de son travail dans Les aveugles: Stéphanie Jasmin, «Parcours du personnage vidéo », Alternatives théâtrales, $\mathrm{n}^{\circ} 73^{-74}$, juillet 2002, p. 40-42. 


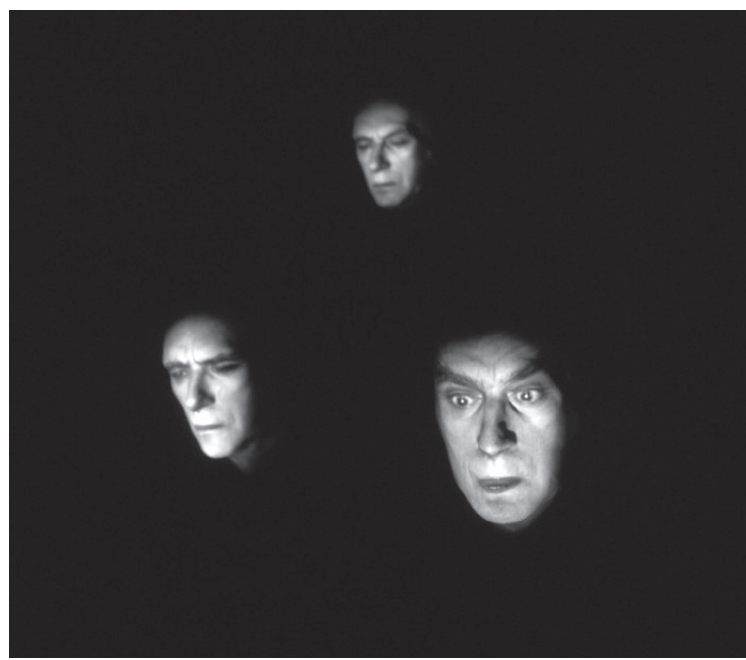

Fig. 1. Les aveugles. Fantasmagorie technologique I, 2002, (C) photo Richard-Max Tremblay (avec l'aimable autorisation d'UBU, compagnie de création).

Malgré l'utilisation de ces technologies de l'image et du son, par ailleurs, le projet reste artisanal, dans la mesure où l'appareillage technologique ne permet pas de reproduire instantanément le spectacle lorsque la compagnie s'installe dans un nouveau lieu. Les techniciens qui investissent l'espace pour installer la «sculpture vidéographique» doivent réajuster manuellement les masques et les projections de manière à ce que l'image reprenne forme dans son volume. Finalement, si l'installation est reproduite de manière identique dans chaque nouvelle salle (la fiche technique est très précise et exigeante quant au type d'espace requis), la balance de son des voix et des effets sonores est à ajuster et à moduler selon la constitution de murs, plafonds et environnements extérieurs à ces espaces.

\section{Perception de la sculpture vidéographique: analogies avec l'art vidéo}

Le spectateur, face aux Aveugles, est confronté à une œuvre se situant entre l'installation et la représentation théâtrale. Denis Marleau a en effet réalisé une œuvre aux allures d'installation, et dans la mesure où le dispositif est constitué de nombreux supports et projections, il est possible de convoquer l'expression de René Berger et de parler de «multividé $0^{26}$ », acception qui correspond aux

26. René Berger, «Les arts technologiques à l'aube du Xxi ${ }^{\mathrm{e}}$ siècle», dans Louise Poissant (dir.), Esthétiques des arts médiatiques, Sainte-Foy, Presses de l'Université du Québec, 1995, tome I, p. 81. 
installations et sculptures intégrant plusieurs composantes hétérogènes, ainsi qu'aux espaces multipolaires qu'elles impliquent. Les aveugles revêt l'aspect d'une sculpture vidéographique, les supports disposés dans l'espace formant un ensemble composite ${ }^{27}$. Toutefois, contrairement à l'installation, Les aveugles implique le spectateur dans un rapport frontal: comme au cinéma ou dans une salle de théâtre à l'italienne, d'un côté se trouvent les supports animés, et de l'autre, les spectateurs, ces derniers ne pouvant ni circuler ni interagir avec eux.

Le dispositif des Aveugles évoque aussi certains procédés du langage vidéographique. Lorsque le vidéaste ajuste les projections sur les masques, «il place le nez à la place du nez, les lèvres entre leurs commissures et les yeux dans les yeu $^{28}$ ", et réalise ce qui se rapproche d'une «surimpression». Selon Philippe Dubois, ce procédé «vise à mettre l'une sur l'autre deux ou plusieurs images, de manière à produire un double effet visuel, d'une part de transparence relative [...] et d'autre part d'épaisseur stratifiée, de sédimentation par couches successives ${ }^{29}$ ». Ici, la surimpression implique la projection d'une image sur un volume (et non le mixage de deux images, ainsi que l'entend Dubois). Comme une seconde peau, la projection vidéo laisse en effet transparaître les formes du support, tout en se superposant, trace visuelle sur empreinte matérielle, sur le masque aux traits identiques. Ce dernier offre son volume à l'image qui s'y incruste, ce qui confère un effet de profondeur, d'épaisseur, et concourt à provoquer l'illusion de réel.

Toutefois, Marleau ne développe pas une esthétique de la discontinuité : il n'a pas réalisé de montage majeur, de changement d'axes, ni de trucage à partir des images saisies, lesquelles sont projetées presque telles quelles sur les masques. Par conséquent, les répliques du texte de Maeterlinck se suivent les unes les autres et si l'enregistrement des voix et des images s'est effectué en douze temps distincts, le caractère fragmenté du processus de création disparaît lors de la représentation. L'image et le son émergent simultanément des projecteurs, mais les dialogues sont présentés selon l'ordre prescrit par l'auteur et produisent une impression de linéarité; le spectateur, bien qu'enveloppé par un environnement sonore complexe, entend clairement le texte de Maeterlinck. Marleau, en effet,

27. Le dispositif rappelle, plus précisément, les œuvres de vidéastes qui travaillent avec la projection vidéo et utilisent divers types de supports - murs, objets, volumes, écrans: Tony Oursler, Bill Viola, Pipilotti Rist, et bien d'autres.

28. Stéphanie Jasmin, «Parcours du personnage vidéo », p. 40.

29. Philippe Dubois, «Vidéo et écriture électronique. La question esthétique », dans Louise Poissant (dir.), Esthétiques des arts médiatiques, p. 163. 


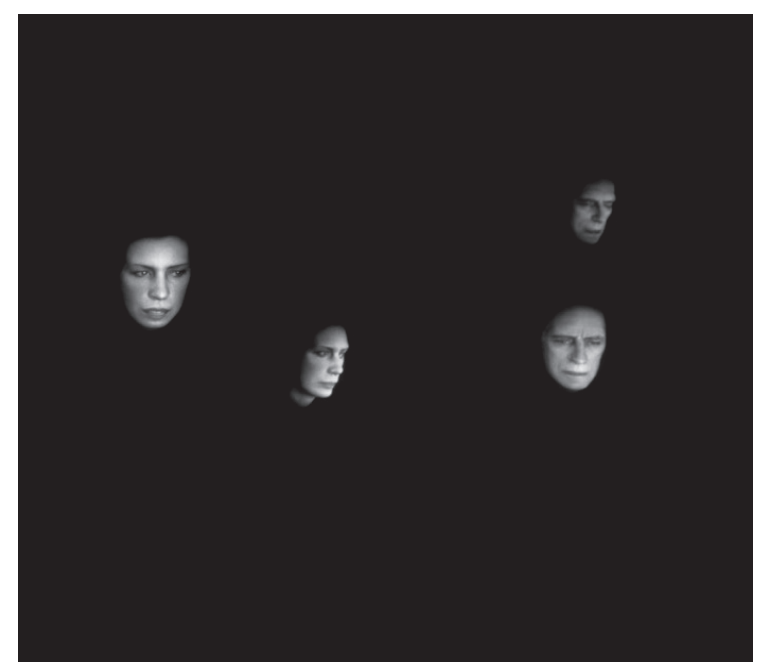

Fig. 2. Les aveugles. Fantasmagorie technologique I, 2002, (C) photo Richard-Max Tremblay (avec l'aimable autorisation d'UBU, compagnie de création).

utilise très peu les effets de superposition des voix et des sons, du moins jamais jusqu'à créer un effet de brouillage ou de discontinuité.

Par ailleurs, puisque l'appareillage technique est dissimulé, les projections vidéographiques instaurent avec le public une relation qui peut s'apparenter à celle du spectateur au cinéma: le code narratif du langage cinématographique «va permettre d'intérioriser l'image, d'oublier durant le temps de la projection la matérialité de l'écran et la nature plate de l'image [...] par l'identification au regard de la caméra $3^{\circ}$. » Le dispositif conçu par Marleau, comme celui de la fantasmagorie, parce qu'il amenuise la frontière entre la scène et la salle, plonge les spectateurs et les masques dans l'obscurité et anime des écrans en relief, travaille justement à créer l'illusion du réel et à effacer les traces de la médiation. Le spectateur se trouve inclus dans le dispositif, immédiatement face à ces étranges figures comme face à des fantômes, là, présents, masques mortuaires incarnant des êtres disparus. Au sujet des Aveugles, dans un article portant sur l'image et la référence au cinéma, Olivier Asselin note que « la rencontre de l'image technique et du moulage [...], qui s'épousent et se donnent vie l'une à l'autre, renforce l'effet

30. Michel Chion, Lart des sons fixés, Fontaine, Éditions Metamkine, Nota Bene, Sono-Concept, 1991, p. 65. 
de réel. » Toutefois, poursuit-il, «l'illusion ne dure pas¹.» Les acteurs, en effet, subissent une « dématérialisation ${ }^{32}$ » puisque, d'une part, on ne voit que leur image filmée, et, d'autre part, on n'aperçoit que leur tête. De surcroît, Marleau démultiplie l'image des acteurs: le personnage perd toute caractérisation individuelle et devient une entité anonyme, à l'instar des personnages de Maeterlinck, qu'il définit lui-même à peine (ils se nomment le cinquième, la jeune, les trois vieilles, etc.). Fragmentée, l'image représente non pas le personnage, mais une figure de l'entre-deux, une représentation éphémère de l'acteur disparu ${ }^{33}$. Ainsi, le dispositif tend à se faire oublier, à devenir transparent, et affiche dans le même mouvement ses conventions et subterfuges, son artificialité34; c'est peut-être cette ambiguïté qui confère à la représentation son caractère étrange et fantasmagorique.

Cette installation-théâtre, parce qu'elle interroge la possibilité de représenter l'invisible, parce que, par le biais de la vidéo, elle met en question la présence de l'acteur, devrait en principe bouleverser la définition de la représentation. Elle le fait effectivement, en suscitant plusieurs questions et en multipliant les ambivalences. Même si les acteurs sont absents de la scène et que la représentation repose sur le dispositif scénique, le travail de Céline Bonnier et de Paul Savoie demeure essentiel dans l'œuvre, dans la mesure où ce sont leur voix et leur visage qui font entendre, portent et interprètent le texte. De plus, malgré l'absence des acteurs, ces derniers sont rendus présents au public par un subterfuge technologique et si leur «qualité de présence» diffère, ils habitent néanmoins toujours l'espace des Aveugles. Le spectateur, quant à lui, après avoir vécu une expérience très particulière, celle d'une grande proximité avec ces visages entre la vie et la mort, et même s'il assiste hic et nunc à une représentation théâtrale, hésite quelques instants à applaudir les masques lumineux, traces d'acteurs à la fois présents et absents, lorsque les voix s'éteignent. Dès lors, jusqu'à quel point, pour revenir au questionnement évoqué plus tôt, la vidéo modifie-t-elle cette représentation

31. Olivier Asselin, «Le fantôme et l'automate. De la reproductibilité technique sur la scène », Alternatives théâtrales, $\mathrm{n}^{\circ}$ 73-74, juillet 2002, p. 28.

32. Olivier Asselin, «Le fantôme et l'automate», p. 25-26.

33. Au sujet de la question de l'effet de présence et de la représentation de la mort, je renvoie à l'article d'Olivier Asselin («Le fantôme et l'automate», p. 24-28) ainsi qu’à un texte que j'ai fait paraître: «Projections de la mort. Sur deux mises en scène de Denis Marleau », L'Annuaire théâtral, $n^{\circ} 37$, printemps 2005, p. 113-127.

34. Ces deux statégies peuvent correspondre aux tendances qu'observent Jay David Bolter et Richard Grusin dans le processus de remédiation, soit l'«immediacy» et l'«hypermediacy» (Remediation. Understanding New Media, Cambridge, Massachussetts, MIT Press, 1999). 


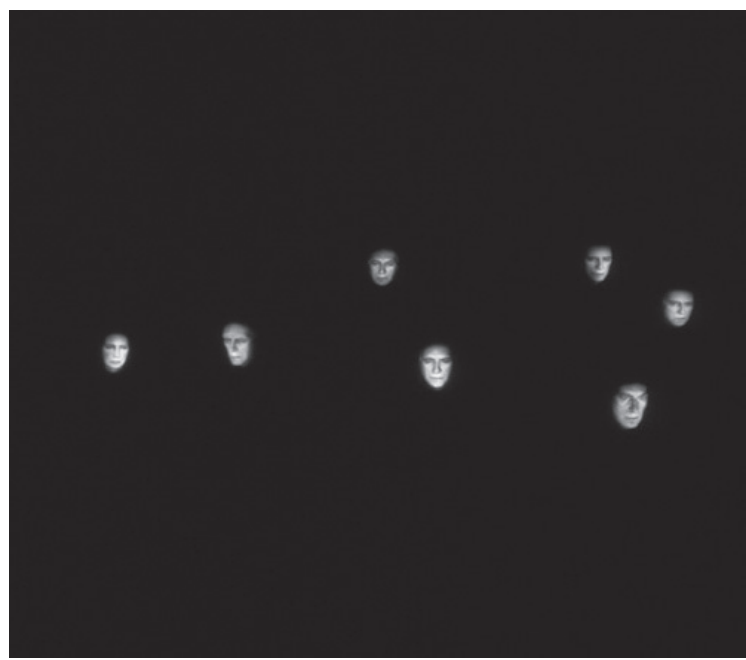

Fig. 3. Les aveugles. Fantasmagorie technologique I, 2002, (C) photo Richard-Max Tremblay (avec l'aimable autorisation d'UBU, compagnie de création).

théâtrale, du point de vue du spectateur? Le médium ne se trouve pas tout simplement juxtaposé dans la représentation, objet autonome dont la fonction serait descriptive, sans souci d'intégration. Denis Marleau, pourrait-on dire, a plutôt extrait la théâtralité de cette discipline non théâtrale; les masques animés ne prennent sens que dans le cadre de la représentation et de la proposition du metteur en scène pour faire entendre le texte de Maeterlinck. La vidéo devient théâtre, de plus, lorsqu'on l'envisage comme un outil (parmi d'autres, aux côté de l'ombre de Maeterlinck et des spectres des fantasmagories), visant, si l'on considère, à l'instar de Monique Borie, la représentation théâtrale «comme un espace où l'invisible trouve sa matérialisation dans le visible de la scène 35 », à donner une forme aux fantômes et aux réalités immatérielles. Cet outil, toutefois, bouleverse la notion de théâtralité en la mettant en question, et en permettant d'élaborer avec le spectateur une relation inédite.

De toute évidence, l'utilisation de la vidéo modifie également la pratique du metteur en scène, qui travaille, à partir d'un certain moment, avec le matériel préenregistré plutôt qu'avec les acteurs vivants. Cependant, saisir véritablement l'impact de l'intégration de ce médium sur la pratique du metteur en scène

35. Monique Borie, Le fantôme ou le théâtre qui doute, Arles, Actes Sud, Académie expérimentale des théâtres, coll. «Le temps du théâtre », 1997, p. 10. 
aurait exigé l'observation, «sur le terrain », de tout le processus de création. Du reste, ce seul travail, déjà considérable, aurait-il été suffisant? Dans la mesure où Marleau explore les ressources de la vidéo depuis la fin des années 1990, ne faudrait-il pas étudier comment, depuis Les trois derniers jours de Fernando Pessoa, la pratique de Marleau a été graduellement influencée, modifiée par le médium? De plus, Les aveugles constitue un jalon dans une trajectoire de création autour de la question du double et du fantôme, dont les origines se situent peut-être même avant Les trois derniers jours..., en ce sens que la marionnette et les ombres apparaissent déjà dans des spectacles des années $1980^{36}$, ou parce que les outils sophistiqués mis en œuvre pour la réalisation de l'environnement sonore sont utilisés pendant les années 1990. Quelles sont les limites du terrain d'observation du chercheur «ethnologue » d'Antoine Hennion, dans le cas d'une démarche théâtrale qui s’inscrit dans la durée, s'échelonne sur plusieurs mois, voire plusieurs années?

Les travaux d'Antoine Hennion impliquent des recherches beaucoup plus vastes que ce qui est proposé ici, puisqu'ils dépassent le processus de création et tiennent compte - il s'agit de l'aspect «ethnographique» de l'approche - de l'ensemble des conditions d'émergence de l'œuvre. Ainsi, en amont et en aval de la production, le fonctionnement de la compagnie et la relation au public peuvent devenir, dans cette perspective, objets de recherche. Dans le cas des Aveugles, par exemple, le fait de créer cette pièce dans un musée plutôt que dans une salle conventionnelle (bien que par la suite, elle ait été présentée, dans plusieurs pays, exclusivement dans des lieux théâtraux), a très certainement participé à modifier l'appréhension du spectacle par le public, à déplacer les conditions de réception. L'approche d'Hennion m’apparaît en somme surtout intéressante pour l'étude de l'objet théâtral dans le contexte de la pratique contemporaine, alors que le métissage des arts sur la scène déplace les enjeux de la représentation. Un travail d'observation "sur le terrain », au sein de plusieurs compagnies théâtrales, permettrait certainement de mieux évaluer dans quelle mesure les frontières entre les arts sont transgressées et quelles sont les conséquences de ces échanges, de différencier les nombreuses pratiques, les multiples manières d'intégrer les autres arts sur les scène de théâtre.

La description succincte du processus et du dispositif, ici, de même que l'esquisse des analogies avec le langage vidéo, ont en effet permis de préciser en

36. Denis Marleau évoque «les ombres, marionnettes et mannequins de l'Ubu cycle (1989) ou des Ubs d'après Jarry, de La victoire sur le soleil (1992) de Kroutchonyck et Malevitch» (Denis Marleau, «Les aveugles et l'utopie», p. 83). 


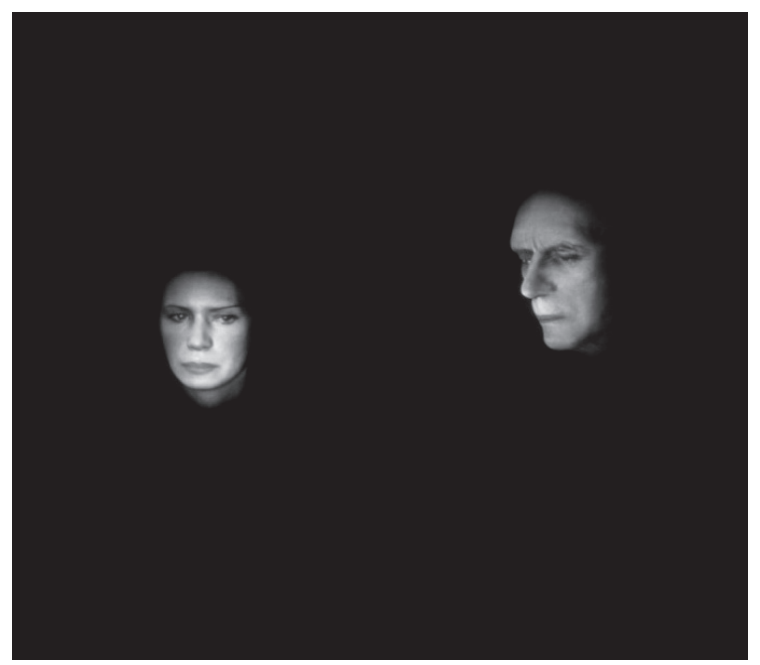

Fig. 4. Les aveugles. Fantasmagorie technologique I, 2002, (C) photo Richard-Max Tremblay (avec l'aimable autorisation d'UBU, compagnie de création).

quoi la théâtralité et la présence de l'acteur sont mises en question, et d'observer les moyens mis en œuvre pour réaliser l'effet de présence. Cette approche m'a toutefois entraînée à départager la forme et le sens de l'œuvre théâtrale, les moyens scéniques et le texte qui, pourtant, «est d'abord ce qui [...] fait rêver le théâtre37 » selon le metteur en scène d'UBU. Après l'étude du dispositif et de son fonctionnement, on ne peut éviter d'envisager la pertinence de cette forme scénique en regard du texte de Maeterlinck et en fonction du cheminement artistique de Denis Marleau, quitte à s'aventurer dans ce que la représentation a de fugitif et que Hennion souhaiterait contenir. L'intérêt des Aveugles, au-delà de l'exploit technique constitué par le dispositif qui pose les questions essentielles de la représentation, réside dans les mots que les acteurs font entendre, dans le potentiel de suggestion que cette nouvelle forme recèle et dans l'expérience, bien qu'éphémère parce que théâtrale, de l'apparition de figures dont l'enchantement s'évanouit lorsque le dispositif s'éteint et se laisse observer.

37. Josette Féral, «Une approche ludique et poétique», p. 183. 